\title{
tendencia editorial
}

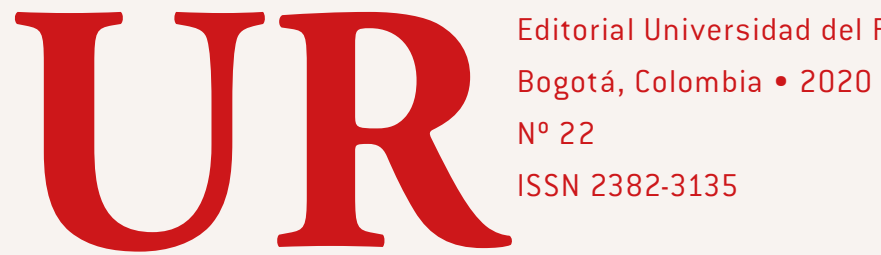

REFLEXIÓN

Vueltas y giros al tornillo sinfín (del mundo)

Rafael Alejandro Nieto Roca

AL ENCUENTRO CON

Repensar el libro.

Estrategias digitales en el tiempo del coronavirus

Entrevista a: María Elvira Jaramillo

Fotogramas a la historia de la edición. Un pasado que se actualiza y conecta mundos siempre diversos Luis Enrique Izquierdo Reyes

ACTUALIDAD

Lo que recomiendan nuestros lectores

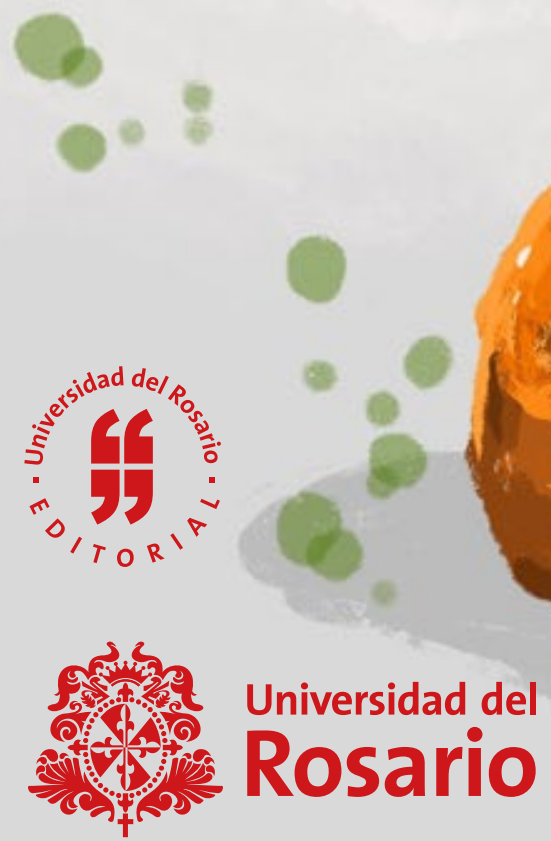


UNIVERSIDAD DEL ROSARIO

MAYO DE 2020

\section{Editorial Universidad del Rosario}

Dirección: Cra.7 \# 12B-41, oficina 501

Teléfono: (57-1) 2970200, ext. 3114

http://editorial.urosario.edu.co

\section{Comité editorial de esta edición}

Universidad del Rosario

Juan Felipe Córdoba Restrepo

Coordinación publicaciones periódicas Tatiana Morales Perdomo

\section{Consejo de edición}

Juan Carlos Ruiz Hurtado

Diego A Garzon-Forero

Libardo Antonio Bernal Castillo

Corrección de estilo

Lina Morales

Diseño, ilustración y fotografía Miguel Gerardo Ramírez Leal Kilka Diseño Gráfico
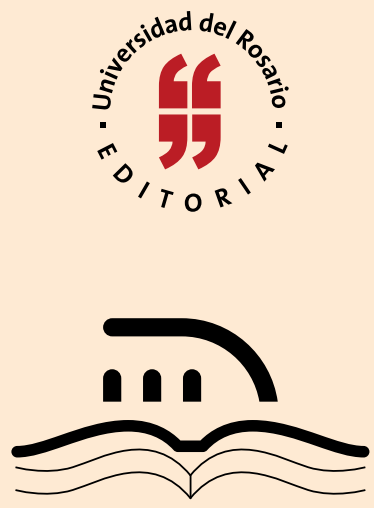

DIFUSIÓN

E D I TO R I A L

U R O S A R I O

\section{REFLEXIÓN}

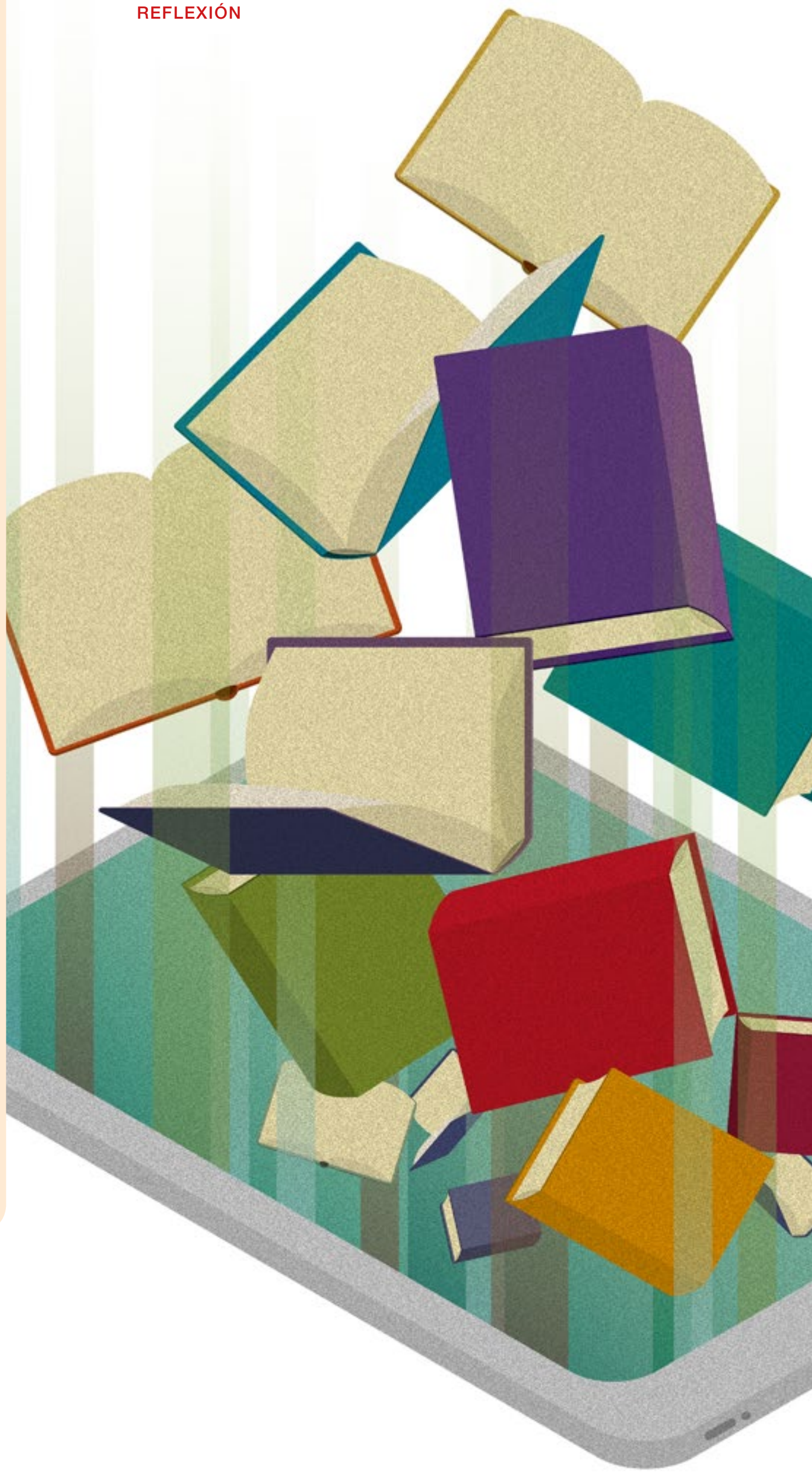




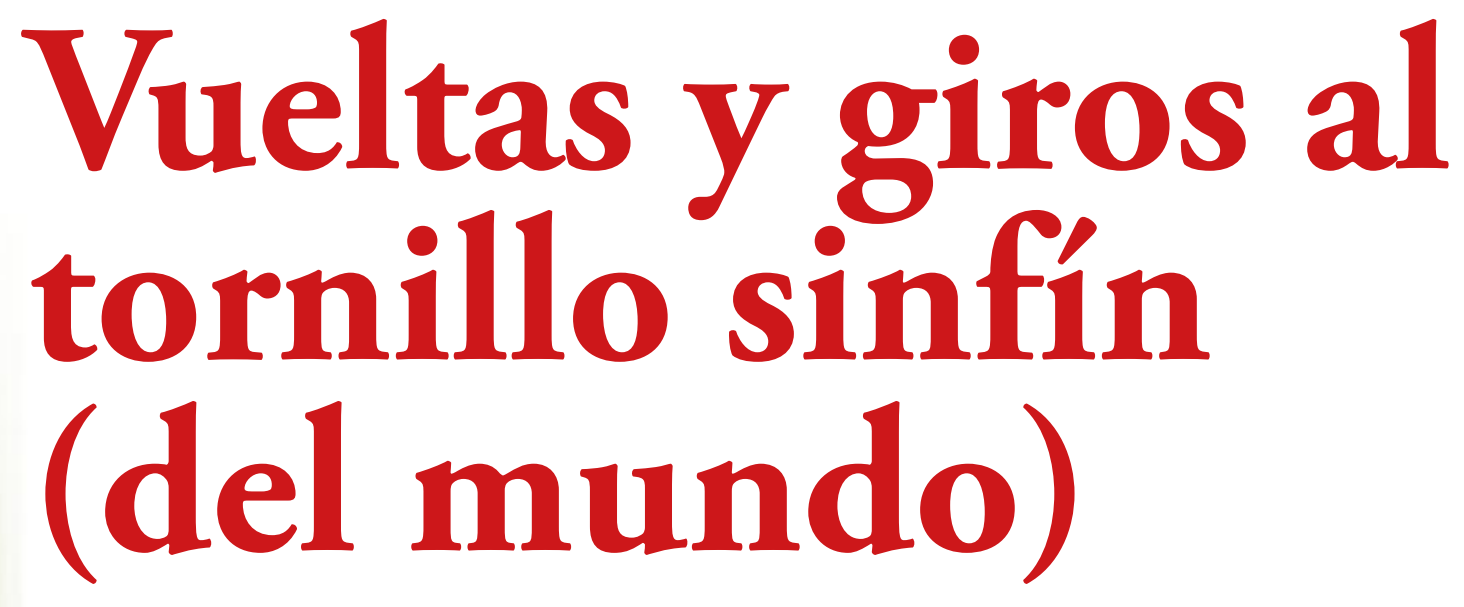

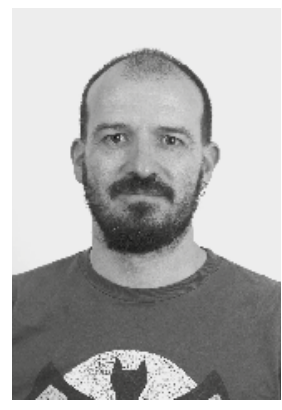

Rafael Alejandro Nieto Roca Coordinador de Promoción y Mercadeo Editorial Pontificia Universidad Javeriana

Para empezar, voy enunciar dos lugares comunes. Primero, el Covid-19 cambió el mundo y, con él, la cotidianidad de gran parte de la población mundial; segundo, las medidas extraordinarias que se emiten en momentos de crisis se convierten en ordinarias al terminar la emergencia. El libro impreso ha sorteado varios hitos en su historia que anunciaban su desaparición.

Un inciso: en textos anteriores recurrí de forma desprevenida a nombrar estas situaciones como mutaciones metafísicas —expresión que acuñó Michel Houellebecq en Las particulas elementales para hablar de sucesos que marcaron un cisma en la historia de la humanidad-, y creo que la situación en la que se encuentra el mundo sí es merecedora de ese apelativo, que antes únicamente usé como una figura literaria. Lo repito: el mundo cambió y depende de la humanidad decidir (o dejar que decidan) la dirección que este giro va a tomar. Fin de mensaje en la botella.

Con la progresión de entrada en rigor de las medidas para atender la emergencia de sanidad, las universidades, $y$, con ellas, las editoriales universitarias, tuvieron que trasladarse a la virtualidad de la noche a la mañana. Aunque mucho del trabajo de divulgación de publicaciones académicas ya se hacía en la red (revistas científicas en acceso abierto y presencia en redes sociales), las oficinas encargadas de la promoción se encontraron, primero, con la imposibilidad de presentar los libros en ferias del libro, eventos académicos en auditorios universitarios o en librerías; y, luego, con toda la cadena del libro impreso clausurada. 


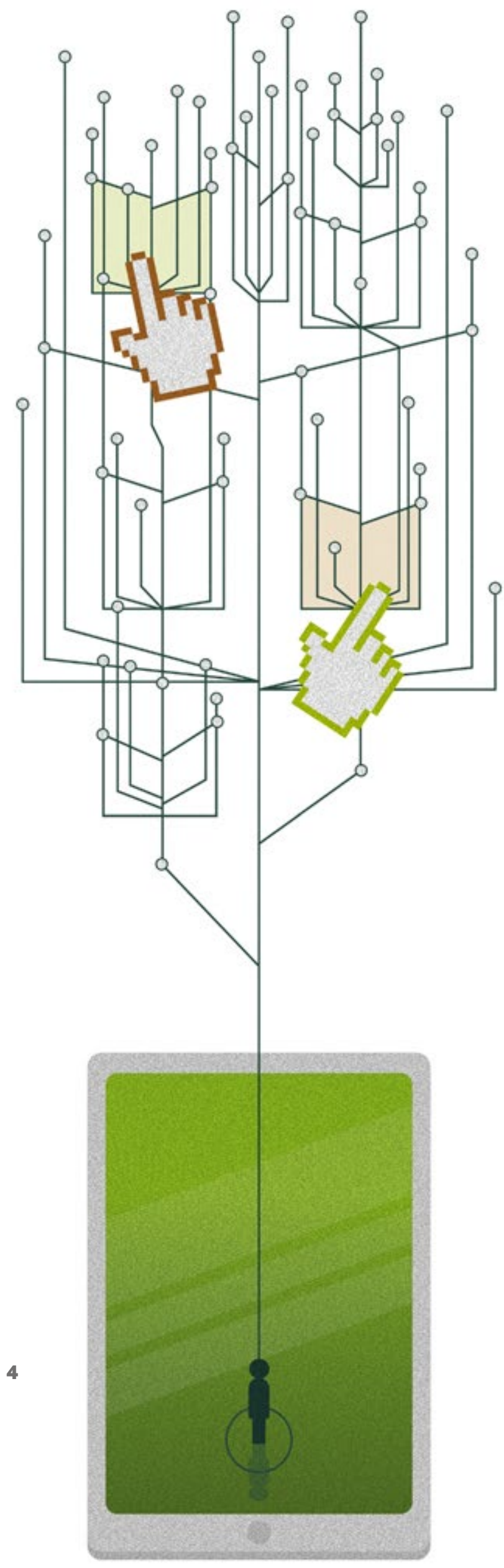

Desde estas oficinas de promoción y mercadeo nos vimos a la tarea de reforzar nuestras iniciativas para divulgar nuestros libros de forma virtual con el fin de promoverlos en diferentes plataformas de visibilidad y comercialización.

Muchas universidades cuentan con una colección especial para alojar sus libros en sus repositorios institucionales. De esa forma, es posible asignarles un código Doi con el que se facilita su citación, trazabilidad y métricas de impacto. En el caso de la Javeriana, estos documentos que se encuentran en el Repositorio están disponibles para libre lectura y descarga por parte de su comunidad educativa; sin embargo, con la intención de apoyar a la comunidad académica y científica, la Universidad —al igual que muchas otras instituciones de educación superior- tomó la decisión de dejar el R. I. en acceso abierto por un tiempo limitado.

Se sigue imprimiendo: las publicaciones en papel se despachan a las librerías para que estén disponibles para compra a través de sus páginas de internet. Tan pronto se publican, diseñamos una pieza de divulgación digital que se envía a bases de datos y se comunica a través de redes de divulgación. Las personas participantes en el proyecto reciben esta pieza para publicarla según lo crean conveniente, convirtiéndose en un eslabón adicional de la red de promoción de su libro.

Con el fin de permitir que los libros en formato impreso tuvieran la posibilidad de circular por librerías, ferias editoriales y bibliotecas, muchas universidades dilataban la conversión a formato .epub por dos o tres meses hasta que el título estuviera consignado en librerías; en este nuevo escenario, este proceso inicia tan pronto como existe el PDF de impresión. Ese archivo .epub se entrega a agregadores digitales para ser distribuido en las plataformas comerciales y las bases de datos con las que tenemos convenio de distribución y visibilidad. En ese momento, se vuelve a enviar un correo similar al que se diseñó para la novedad impresa y se sigue el mismo procedimiento.

La coyuntura nos ha llevado a repensar nuestros actuales proyectos de divulgación y a desarrollar unos nuevos. Todos están diseñados para promover nuestras publicaciones y llevar mayor tráfico a los portales de las editoriales universitarias, pero también se enfocan en la creación de comunidades interesadas en compartir y divulgar conocimiento juicioso en una época en la que la desinformación campea por la red de forma impune.

En el caso concreto de la Javeriana, desde hace seis años contamos con una iniciativa llamada booktrailers, videos que la Editorial produce en asocio con el Centro Ático con la que se realizan cuatro cortos audiovisuales por semestre en los que destacamos las novedades principales. Las piezas tienen gran trabajo de preproducción, construcción narrativa y edición; cada conjunto se toma alrededor de tres meses para salir al aire. El aprendizaje es que en esta temporada se ha innovado en las modalidades de grabación remota y en los tiempos de entrega del material. Igualmente, ha sido necesario instruir a autoras y autores en cómo manipular 
equipos de grabación audiovisual caseros, algo que debió ser de mucha utilidad para la virtualización de sus cátedras y otros aspectos clave de su trabajo. Por otro lado, hace dos semanas creamos un modelo más liviano de cortos audiovisuales para promover y visibilizar nuestros libros; lo estrenamos con la idea de producir un video semanalmente. Se trata de piezas mucho más escuetas y concisas: muy diferentes en su naturaleza y estilo narrativo a los booktrailers.

Contamos con una revista digital, Impronta, en la que publicamos artículos cortos que redactamos para reseñar y destacar nuestros libros. Algunos de ellos se publican primero en la versión impresa de la revista institucional Hoy en la Javeriana, que luego enriquecemos con contenido interactivo para publicar en la red. Impronta tuvo un cambio en su diagramación este año para mejorar su lecturabilidad. Además, se alimentará de las nuevas iniciativas para articular piezas de periodismo digital multimedial, interactivo y dinámico.

Aprovechamos la coyuntura para revivir nuestros podcasts. Tenemos dos proyectos en curso. El primero, Impronta sonora, es un documento sonoro que busca hacer un desglose de un libro y una retrospección del proyecto de investigación o reflexión académica de su autora o autor. Lo pensamos con un estilo narrativo complejo que requiere el apoyo conceptual y de edición sonora de Centro Ático. El segundo, Contraforma, es una entrevista corta de cuatro preguntas y se emitirá semanalmente. Su estilo sobrio abre la posibilidad de grabarlo y transmitirlo en vivo para luego publicarlo en plataformas como Spotify, Pocket Casts y Apple Podcasts. La idea es que estos documentos, además de alimentar Impronta, no se limiten al público universitario y mejoren el impacto de las publicaciones.

Un grupo de comunicadores y comunicadoras de editoriales universitarias y académicas conformó una red de trabajo llamada Conversa. Desde julio del año pasado programa eventos en librerías en los que dos o tres docentes, moderados por un periodista, establecen una conversación en torno a un tema de coyuntura y bajo un postulado definido. Este evento abrió la puerta para destacar una curaduría temática especializada en el tema central, compuesta por títulos de nuestros fondos. Esta curaduría se publica con un artículo corto que invita al evento y que circula de forma conjunta en las redes de las siete editoriales. Con la nueva coyuntura, la red está trabajando en trasladar estos eventos a espacios virtuales a través de diferentes plataformas. Por su parte, el gremio editorial se ha embarcado en desarrollar nuevas experiencias en la red: durante el período en el que se habría desarrollado la Feria Internacional del Libro de Bogotá, el país contará con tres festivales virtuales que nacen gracias a iniciativas paralelas de la Cámara Colombiana del Libro, un grupo de editoriales independientes y un agregador digital de contenido académico.

Esta coyuntura nos está dando la oportunidad de enriquecer algunos de nuestros proyectos de divulgación, así como afianzar nuestras relaciones con medios internos de nuestras universidades. El impacto de la crisis ha llevado a los medios tradicionales a acudir con mayor frecuencia a expertos de la academia que puedan dar respuestas claras a su audiencia. Todas estas iniciativas se suman a las ya tradicionales: entrevistas que se transmiten en los programas de las emisoras universitarias, las páginas de reseñas en medios especializados y tradicionales, y, por supuesto, nuestra programación de los eventos que se realizan en la Universidad, en librerías y en ferias regionales y nacionales, que, a mediano o largo plazo, regresarán progresivamente a hacer parte de nuestro quehacer de divulgación. Quiero retomar una reflexión que hice en el inicio de este texto: de nosotras y nosotros depende el giro de la tuerca que dará este momento histórico. No creo, como ya he oído y leído en diferentes espacios —en el que algunos talibanes de la edición electrónica retoman las banderas raídas que enarbolaron hace quince años_-, que el Covid-19 marque el fin del libro impreso y su canal de distribución, como no creo que este fenómeno sea el principio del fin de las relaciones humanas fuera de la red y nos confine a nuestras personas electrónicas. Habrá cambios, por supuesto: puede que algunos actores —incluso, algunos eslabones en la cadena del libro- desaparezcan o se transformen, pero el gremio editorial y librero a lo yente y resiliente. 


\section{REPENSAR}

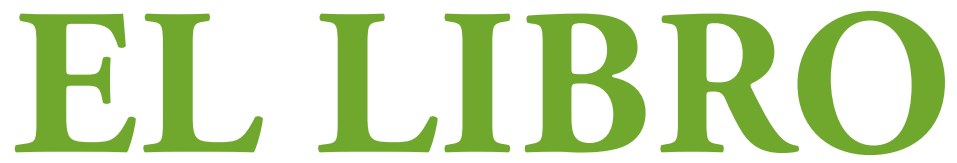

\section{Estrategias digitales en el tiempo del coronavirus}

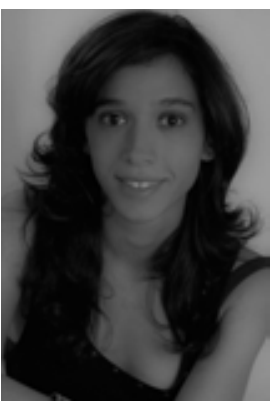

María Elvira Jaramillo

Directora de

Comunicaciones

Siglo del Hombre Editores 


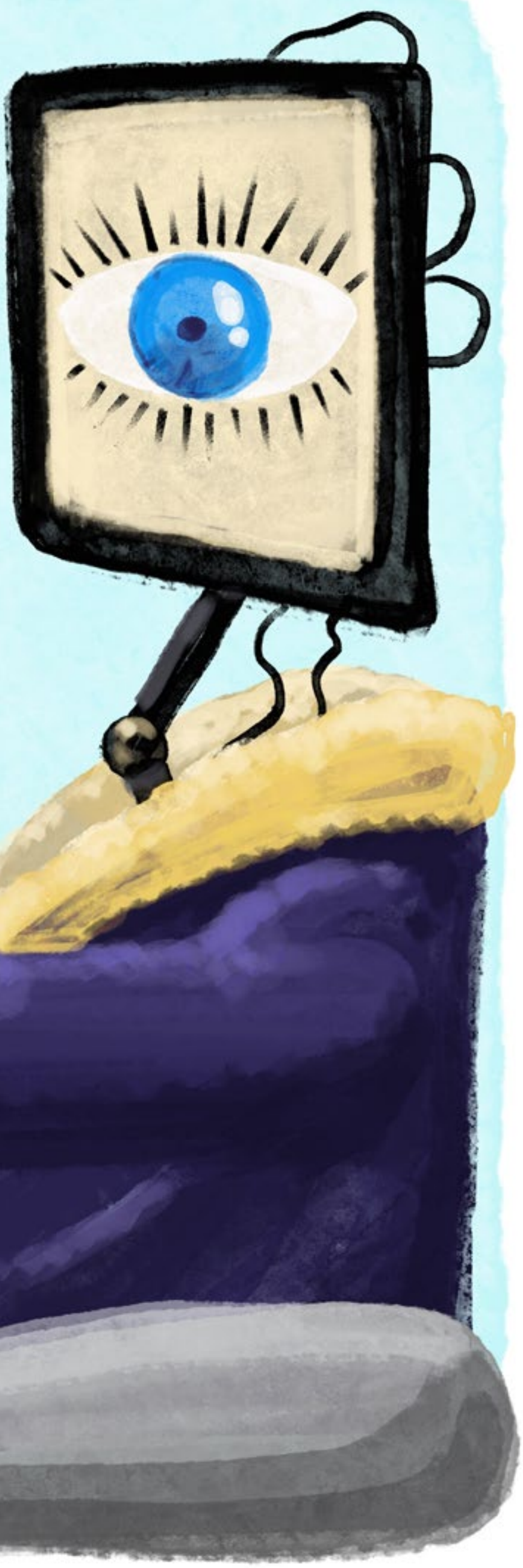

Entender que la situación que afrontamos el día de hoy puede ser algo que se repita en los próximos años, dada nuestra relación con el medio ambiente y el manejo que hacemos de los recursos, nos lleva a replantear muchas cosas. Como editorial comprendemos que los retos que se avecinan para la producción, comercialización y difusión de libros, sobre todo físicos, son bastantes. Principalmente, porque esta situación resucita el viejo debate sobre el valor que tienen las librerías, editoriales y distribuidoras en un mundo globalizado, cada día más digital. Pareciera entonces que el futuro de los libros y de todo lo que está detrás de su elaboración es incierto.

Sin embargo, como ha pasado en otros momentos de la historia, los libros, las librerías y editoriales están afrontado estas circunstancias de una manera loable, que seguro pasará a la historia. Han dejado de lado los límites que las medidas restrictivas para combatir el coronavirus han impuesto en gran parte del mundo. Han innovado, creado nuevas estrategias, nuevos formatos y contenidos, por lo cual como editorial quisimos abrir un espacio para socializar y compartir el trabajo que han desarrollado las principales librerías y editoriales del país. Para esta ocasión, nuestro boletín Tendencia Editorial de la Universidad del Rosario se reunió con María Elvira Jaramillo, directora de Comunicaciones de Siglo del Hombre Editores.

Boletín Tendencia Editorial (BTE): ¿¿De qué manera

Siglo del Hombre se ha visto afectado por las medidas que ha tomado el gobierno para poder prevenir y afrontar la pandemia del coronavirus?

María Elvira Jaramillo (MEJ): Dado que Siglo del Hombre es distribuidor, editorial y librería a la vez, cada línea de trabajo se ha visto afectada de distintas formas. La editorial, por ejemplo, no ha podido continuar con el catálogo de publicaciones y novedades que tenía previsto. Tanto así que no pudimos realizar los lanzamientos planeados para la Feria del Libro de Bogotá. La librería, por otro lado, dadas las circunstancias, lleva cerrada más de cuatro semanas.

En cuanto a la línea de trabajo de distribución, nos hemos visto afectados desde los canales de comercialización. Ahora, la distribuidora no cuenta con su principal canal, la red de librerías en Colombia, lo que ha sido un reto bastante grande para la comercialización y divulgación de libros. No obstante, a través de nuestra tienda virtual, hemos y seguiremos despachando libros.

BTE: Creo que algo interesante de Siglo del Hombre es que contemplaba, incluso antes de esta situación, estrategias y contenido enfocados a los formatos digitales. ¿Podría contarnos un poco más acerca de esta área de trabajo y, específicamente, qué estrategias han tomado en las circunstancias actuales? 
MEJ: Claro, nosotros en Siglo del Hombre tenemos, desde hace seis años, un Departamento de Proyectos Digitales, que se encarga, puntualmente, de tres tareas. La primera, hacer que todo el catálogo de nuestra editorial esté disponible en diferentes versiones digitales y en distintas plataformas de descargas de e-books.

La segunda línea de trabajo de este departamento es subir todas las novedades y mantener actualizado el catálogo de todas las editoriales que tenemos en distribución. Nosotros contamos actualmente con editoriales nacionales e internacionales para distribución digital. Eso quiere decir que subimos contenido a Amazon, a Casa del Libro, a Google Books y a Apple Books, todo el tiempo.

De igual forma, permanentemente estamos buscando otro tipo de alternativas, porque, además de las plataformas comerciales, están las plataformas de bibliotecas, que, hoy en día, también dada la situación, son un boom. Hay una gran cantidad de bibliotecas que abrieron sus catálogos y los dejaron de acceso abierto.

En cuanto a la tercera línea de trabajo, este departamento tiene un objetivo muy de la mano de la pedagogía. En las últimas semanas esta dependencia ha hecho un trabajo muy cercano a los editores, explicándoles qué es un proyecto digital, qué es un $e$-book, qué es un e-commerce y en sí todas las condiciones comerciales y digitales que se han abierto y potencializado dada la situación.

Aunque ya había muchas editoriales que en este momento estaban preparadas para lidiar con las restricciones y los retos en la producción, comercialización y divulgación de libros, muchas otras no habían considerado que era importante efectuar un trabajo desde el área digital. No solo porque es un proyecto muy costoso, sino porque, para muchas editoriales y librerías, esta área no es comparable con el éxito de ventas de libros impresos. Sin embargo, aquellas editoriales o librerías, como nosotros, que ya contaban con catálogos digitales siguieron trabajando, sobre todo durante las primeras semanas, donde no se podían hacer ni siquiera despachos físicos de libros.

BTE: Y en cuanto al área de comunicaciones, ¿cuáles

han sido esas estrategias u objetivos?

MEJ: Creo que es importante que entendamos que esta situación nos está abriendo varias puertas para una nueva forma de trabajo desde las editoriales, desde las librerías y desde los distribuidores, sobre todo desde el área de comunicación. Hoy en día, podemos encontrarnos con muchos más canales de difusión a través de los formatos digitales. Simplemente hay que fortalecerlos, dinamizarlos, activarlos, mejorarlos y seguir trabajando. Nunca parar.

Específicamente, Siglo del Hombre, desde el área de comunicaciones, ha desarrollado e implementado dos objetivos fundamentales. El primero, ajustar e implementar una estrategia de comunicación, de distribución y de divulgación de libros, tanto impresos como digitales. Libros

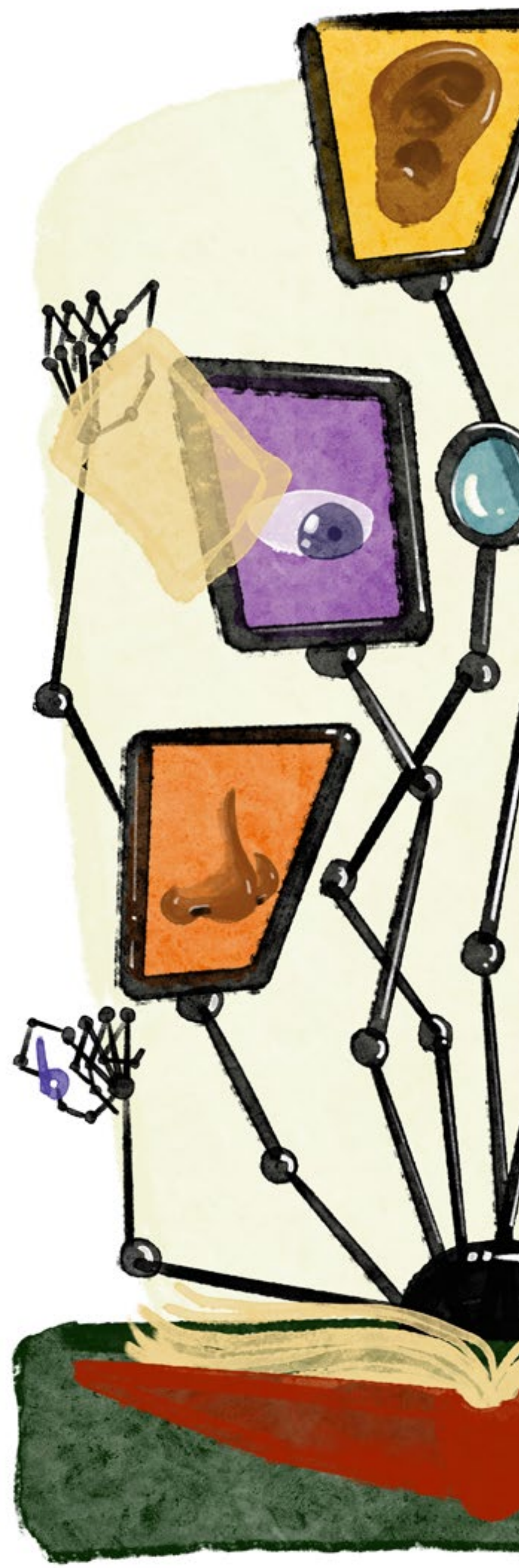




\section{.}
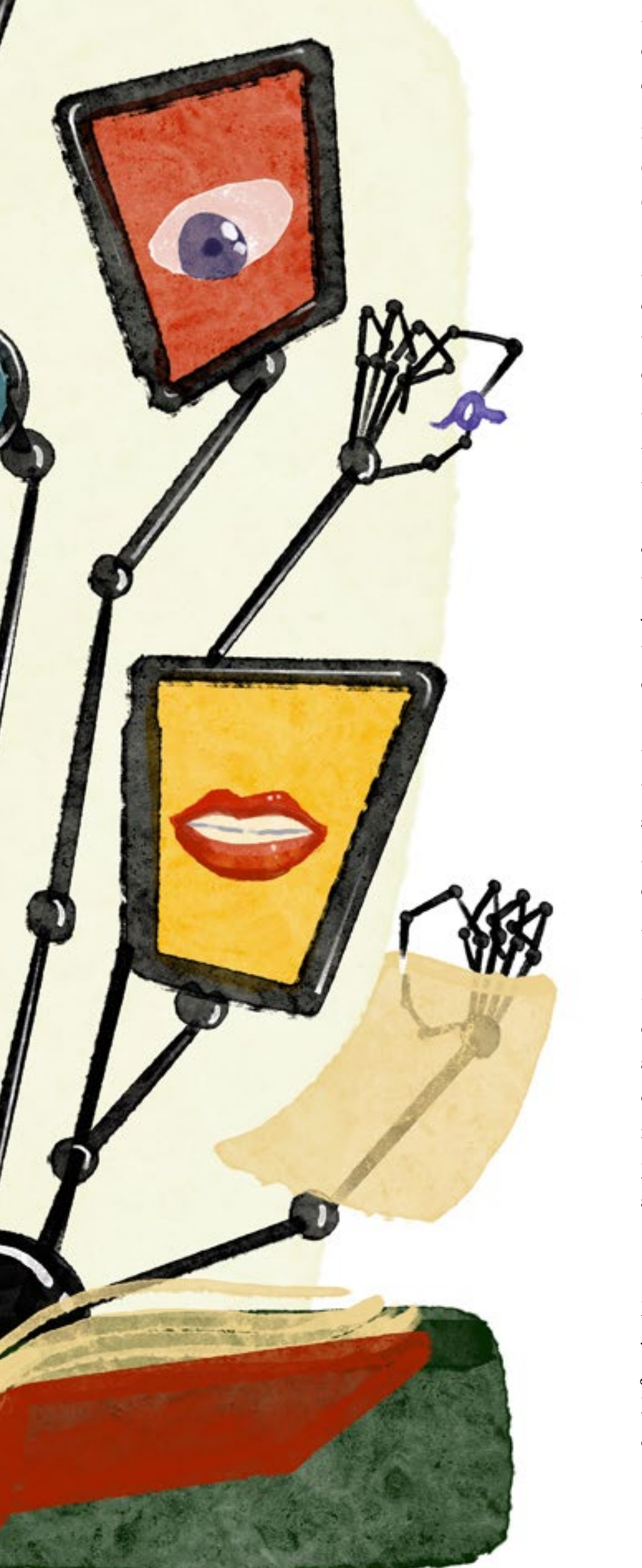
(1) 6 $\frac{1}{0}$

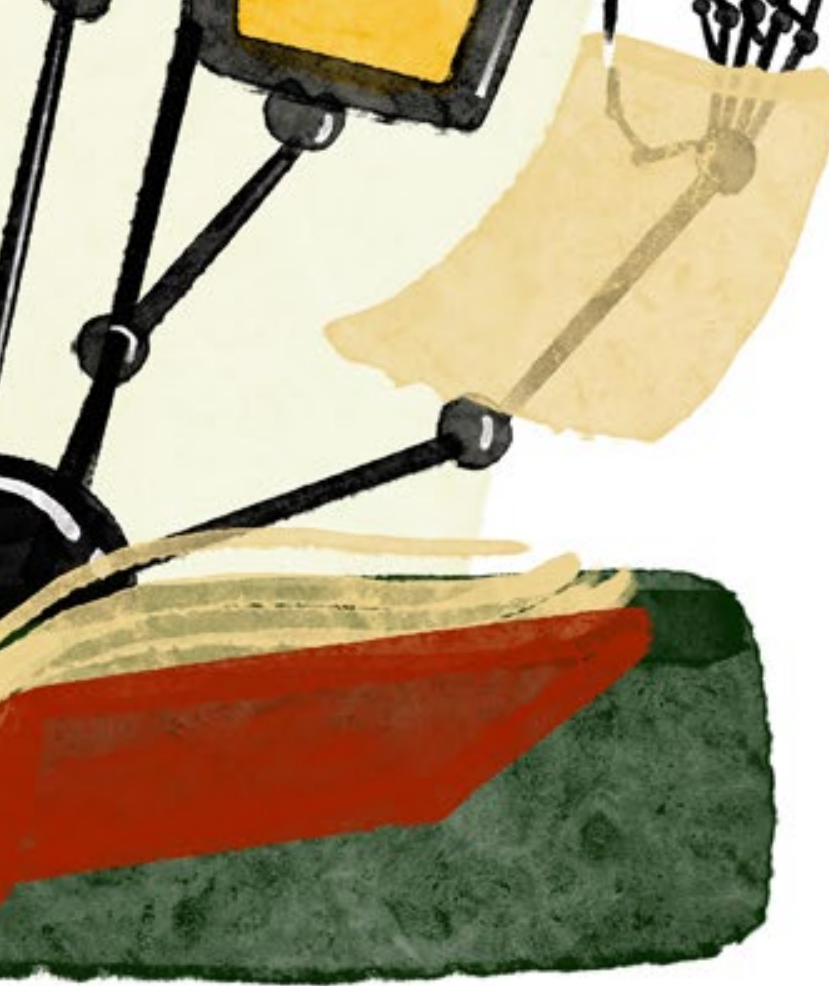

(20) 
BTE: A partir de este tipo de estrategias que han desarrollado, tanto desde el Departamento

de Proyectos Digitales como desde el área

de comunicación, ¿̨cuál diría usted que

es el reto más importante que han tenido

que abordar las editoriales, librerías y

distribuidoras del país? Y, respecto a ese reto, ¿cómo Siglo del Hombre lo ha enfrentado?

MEJ: Pienso que esta situación, al reavivar la discusión sobre el acceso al libro y sobre el $e$-book como gran alternativa de circulación de contenidos, está dejando de lado un tema muy relevante para las editoriales, librerías y distribuidoras. Considero que muchas de estas entidades se han saltado un asunto importantísimo: decirle a la gente qué es un $e$-book, cómo se lee, cómo se accede o se compra este, cómo se entra a una biblioteca digital y se descargan este tipo de contenidos, etc.

Fueron varias editoriales, librerías y distribuidoras las que asumieron que todo el mundo, por lo menos una vez en su vida, había leído un libro en formato digital. Y tú realmente preguntas y el porcentaje de gente que ha accedido a este tipo de contenido no es muy grande. Para muchos, esta es o será su primera vez accediendo a e-books y creo que el reto al que nos enfrentamos como editoriales es que nadie está entendiendo a su público.

Dicho esto, desde Siglo del Hombre, decidimos realizar dos campańas que, en lo personal, me parecieron muy bonitas. Las dos con un valor pedagógico muy importante y con el objetivo de acompañar a lectores y editores en todo el proceso y, sobre todo, en toda la experiencia de lectura de un e-book y la compra en plataformas digitales.

La primera campaña fue "libreriasiglo.com, tu brújula más allá del papel”. Esta campaña tenía como objetivo mostrar el paso a paso de una compra, de una búsqueda, de la lectura y descarga de un e-book, así como dar respuesta a preguntas frecuentes al respecto. También nos interesamos en efectuar y sugerir rutas temáticas de acuerdo con los intereses de los lectores, al igual que una guía de descarga para poder acceder a este tipo de contenido digital. Para muchas personas, todo esto puede parecer muy evidente, pero, si tú oyes a tus lectores y usuarios, te das cuenta de que no lo es.

Por otro lado, la segunda campaña, "Llegó el momento de acceder al mundo digital", tenía como obje- tivo mostrar todos los lugares a donde llegaba nuestra distribución digital y hablar de los canales de acceso al e-book y las nuevas editoriales que teníamos para descarga. Nuestro fin era mostrarles a las editoriales, a las personas y a los lectores que los límites realmente no existían, porque los libros, independientemente de las circunstancias, llegaban hasta muchos lugares. Entonces, hicimos una noticia explicando a qué lugares del mundo llegaban, en qué bibliotecas y librerías online estábamos y cuáles eran las nuevas editoriales que se habían sumado a esta tendencia. Para acompañar esta campaña hicimos la selección de 25 libros para empezar a leer $e$-books.

BTE: Ya para finalizar, ¿cuáles serían esas reflexiones o recomendaciones claves que deben tener en cuenta editoriales, librerías y distribuidores para lidiar con toda esta situación?

MEJ: Lo primero, que ya había mencionado y no me canso de repetirlo, es superar rápidamente la tusa de todo lo que soñamos y no fue. Debemos comenzar a trabajar desde el momento cero. Debemos estar todos los días pensando en los nuevos canales que tenemos disponibles. No en lo viejo, no en cómo se hacían las cosas, sino en lo que tienes en este momento, con qué puedo trabajar. Tengo que llevar todo a otro nivel, porque cada vez más el público, la audiencia, va a ser más exigente. No se va a conformar solo con que publiques una vez en redes o con el hecho de que tengas una página que venda bien; ellos te van a pedir cada vez más y más.

Dos, la recomendación que para mí es la más importante de todas es escoger y conformar un equipo de alianzas fabuloso. Es muy difícil que empresas del tamaño del Siglo del Hombre, que editoriales pequeñas, que proyectos que recién están empezando puedan lograr todo lo anterior solos. Uno tiene que trabajar en alianzas con otras personas, con otras entidades. Esto a nosotros nos da muy buenos resultados. Por ejemplo, en la creación de espacios de divulgación sería imposible pensarnos nosotros sin la red Conversa, una red de varias universidades entre las que se encuentran la Universidad del Rosario, la Universidad de los Andes y la Universidad Santo Tomás, por mencionar algunas. Ahora, todos nosotros estamos trabajando en red para poder sacar eventos muy buenos en el marco de lo que sería la FILBo. 


\section{Fotogramas a la historia de la edición}

Un pasado que se actualiza y conecta mundos siempre diversos

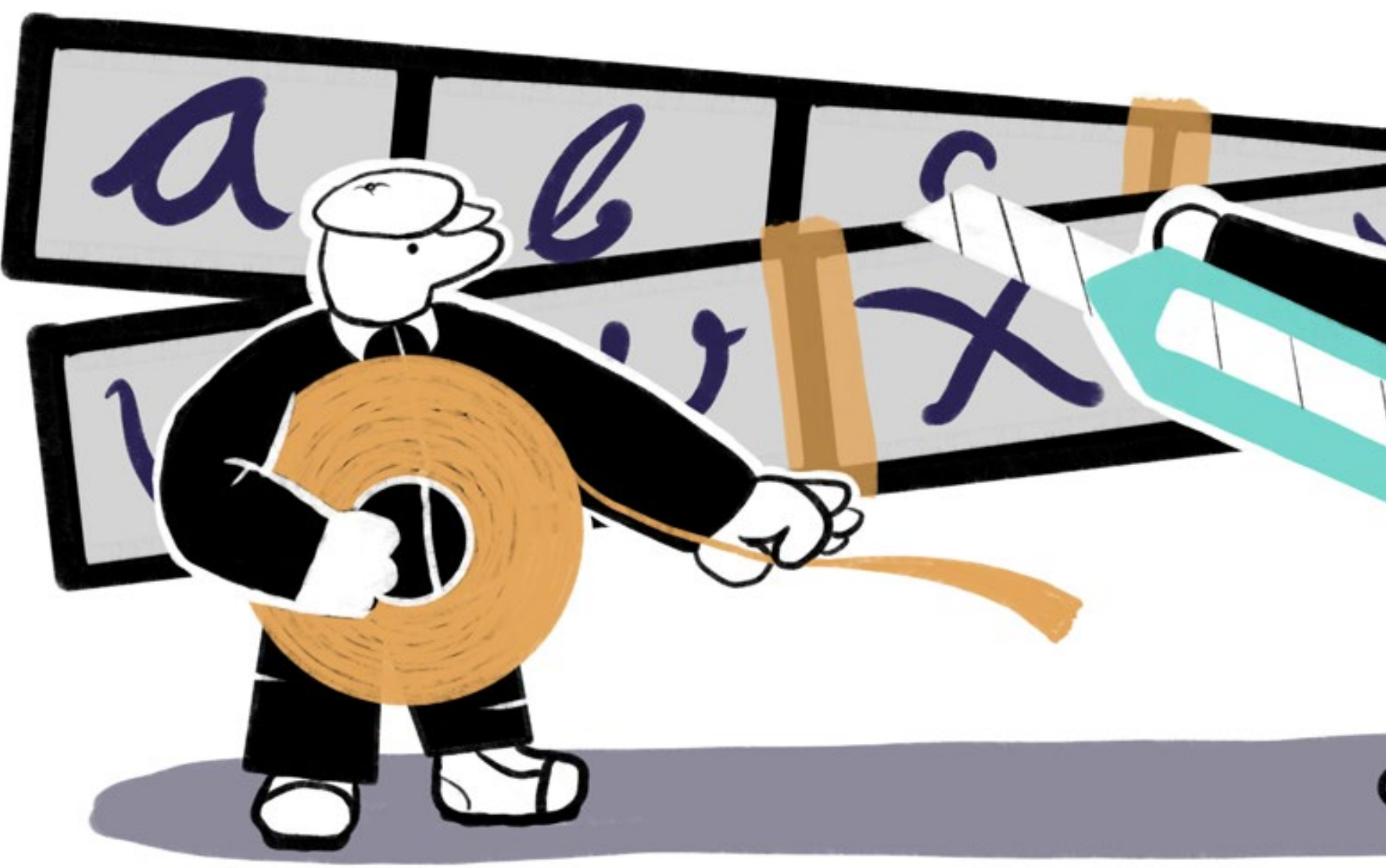




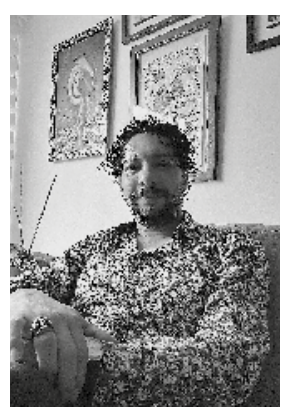

\section{Luis Enrique} Izquierdo Reyes

Gestor cultural y docente de la Universidad del Rosario. Coordinador académico de la Universidad del Sinú Bogotá.

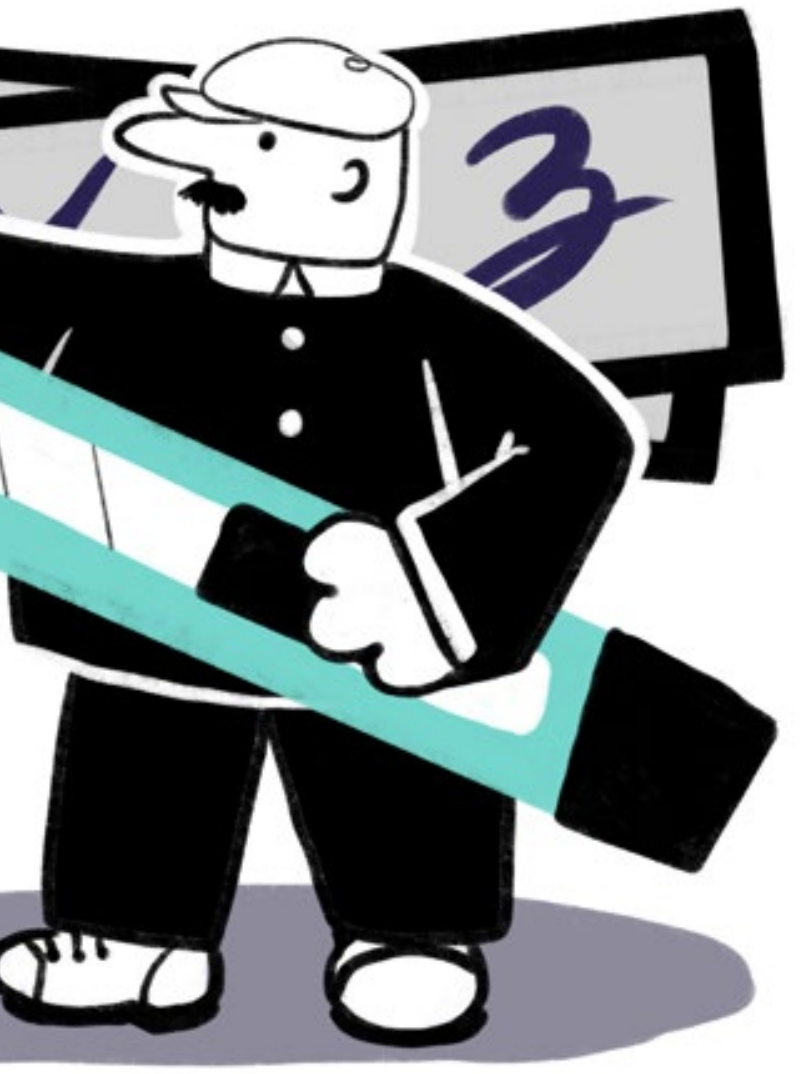

Aventurarse al oficio del editor en un país como Colombia no es ni ha sido una tarea fácil. Desde hace un poco más de diez años ha aparecido en el escenario colombiano una serie de emprendimientos que con tenacidad y mucho corazón ha podido hacerse un lugar dentro del ecosistema editorial nacional. Estas empresas editoriales han combinado, por un lado, el deseo de gestionar nuevos autores, la creatividad en los diseños, el uso de multiplataformas para sus estrategias de mercadeo, el diseño de nuevos productos, así como la elaboración de portafolios de servicios editoriales.

La profesionalización del editor, al igual que la de los oficios y profesiones asociados, tales como diagramadores, correctores, diseñadores, fotógrafos, ilustradores, ha permitido que se entienda la labor editorial como un negocio con el cual es factible vivir y, al mismo tiempo, generar un impacto cultural y de desarrollo en el ámbito en el cual se recrea su trabajo.

Lo anterior, por supuesto, no puede ser ajeno a los acontecimientos históricos, a los procesos que con anterioridad abrieron la brecha para que se gestara una cultura editorial y librera en Colombia. Es así como dentro del ya consolidado catálogo de la Editorial de la Universidad del Rosario aparece un libro que ayuda a entender el fenómeno editorial en el país, desde la perspectiva de aquellos que se empecinaron con dar a la nación elementos para su configuración y consolidación a través de la cultura; en ese sentido surge la obra de Paula Andrea Marín Colorado, Un periodo en la historia de la edición y de la lectura en Colombia (1925-1954). En esta publicación, como si se tratara de un análisis de fotografías, la autora nos transporta a tres momentos de la edición: la aparición de Ediciones Colombia, proyecto de Germán Arciniegas; el proyecto editorial Arturo Zapata; y el desarrollo de la Biblioteca Popular de Cultura Colombiana. De esta manera no solo se fortaleció la edición en Colombia, sino que se desarrolló un catálogo de autores y de temas que terminó por influir en la dinámica cultural del país.

De tal forma, adentrarse en un momento de la historia permite encontrar los vínculos que unen un lado y otro de ella. Los nuevos proyectos de algún modo se conectan con el ímpetu y la visión de dos hombres que a principios del siglo $\mathrm{xx}$ se arriesgaron a proyectos culturales de gran envergadura y construyeron el destino del panorama cultural que tenemos hoy en día. Así que al mirar esas nuevas editoriales podremos ver los ojos de Arciniegas y Zapata desde ese pasado que se actualiza constantemente. 


\section{Lo que recomiendan nuestros lectores}

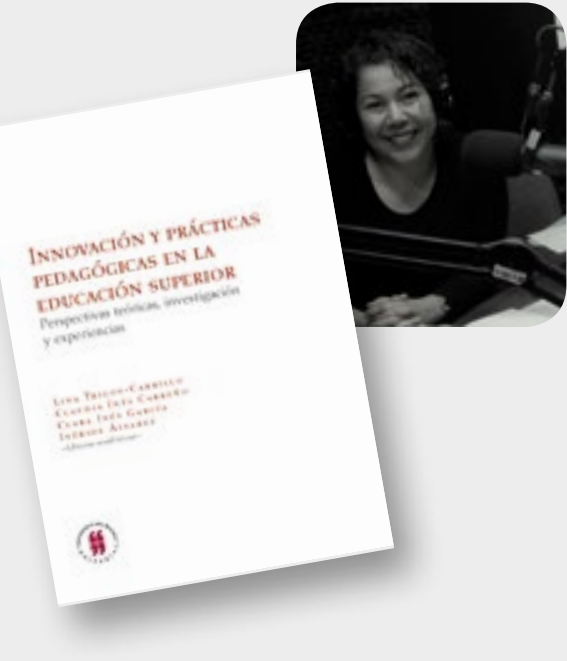

\section{Inéride Álvarez Suescún}

Profesional de Innovación Pedagógica, Dirección Académica del Centro de

Enseñanza, Aprendizaje y Trayectoria Profesoral, Universidad del Rosario

Recomendado: Innovación y prácticas pedagógicas en la educación

superior. Perspectivas teóricas, investigación y experiencias

Autoras: Lina Trigos Carrillo, Claudia Inés Carreño Durán, Clara Inés García e Inéride Álvarez

Mi recomendado es el libro Innovación y de los retos que enfrenta la Universidad del prácticas pedagógicas en la educación superior. Perspectivas teóricas, investigación $y$ experiencias, porque presenta experiencias y reflexiones sobre la innovación de las prácticas pedagógicas en las universidades.

El tema, además, está vigente a propósito

Rosario en la actualidad. Razón por la cual cada uno de los capítulos se constituye en un aporte a las discusiones teóricas y prácticas alrededor de la enseñanza y el aprendizaje en la educación superior en Colombia y América Latina.

\section{Diana Carolina García Sánchez}

Auxiliar de Gestión de Profesores del Centro de Enseñanza, Aprendizaje y Trayectoria Profesoral, Universidad del Rosario Recomendado: En diálogo con la tierra. Por una Colombia sostenible Autores: Diego Pérez Medina y Sebastián Restrepo

Yo recomiendo la obra En diálogo con la tierra. Por una Colombia sostenible, porque expone una perspectiva bastante interesante y amplia sobre el desarrollo sostenible de nuestra nación. Un país con una riqueza natural, ambiental y cultural tan única y diversa que merece ser un referente para el cuidado, consciencia y sensibilización del medio ambiente. Así mismo, al ser un libro con fotografías sobre la diversidad natural y cultural de nuestro país, es ideal para tener y compartir en familia.

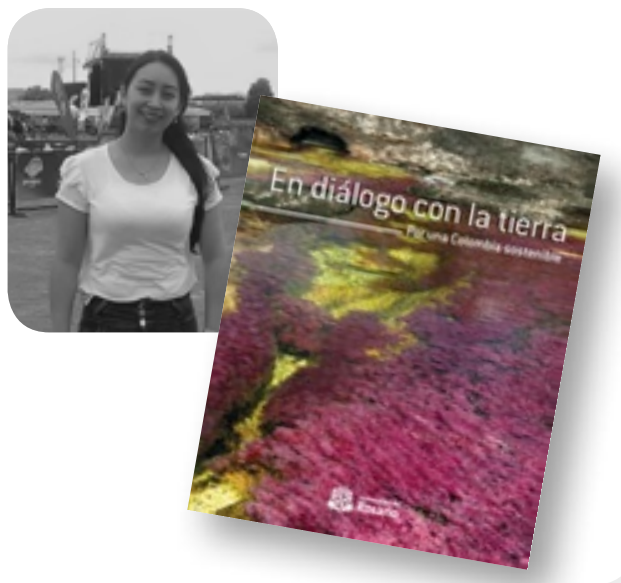

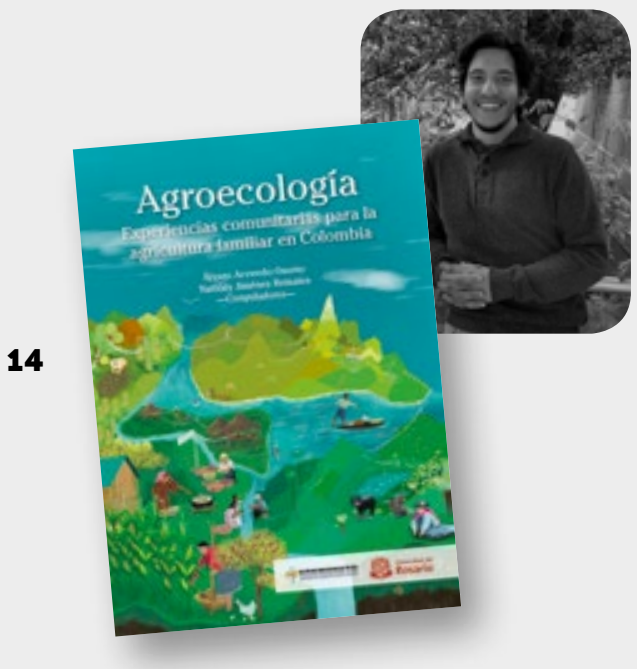

tendencia editorial UR $-\mathrm{N} .^{\circ} 22$

\section{Juan Pablo Mongui Torres}

Biólogo. Estudiante de la Maestría en Ciencias Naturales, Universidad del Rosario Recomendado: Agroecología. Experiencias comunitarias para la agricultura familiar en Colombia Compiladores: Álvaro Acevedo Osorio y Nathaly Jiménez Reinales

Recomiendo el texto Agroecología. Experiencias comunitarias para la agricultura familiar en Colombia, ya que me parece un tema muy interesante para un país como el nuestro, en donde la gran mayoría de terrenos, además de ser aptos para cultivar todo tipo de productos, tienen las condiciones ambientales y productivas necesarias para incentivar los procesos de conservación del medio ambiente y el desarrollo rural sostenible. Este libro, adicionalmente, es muy interesante, porque les permite a los lectores familiarizarse con términos científicos y sociales que, desde las ciencias naturales, se están utilizando mucho en el ámbito de la conservación ecológica. A manera de ejemplo, términos como servicios ecosistémicos y sistemas agroalimentarios. 
Estudiante de Ciencia Política y Gobierno,

Universidad del Rosario

Recomendado: Historia de la primera

República de Colombia, 1819-1831. “Decid

Colombia sea, y Colombia será"

Autor: Armando Martínez Garnica

La historia es considerada como una de las ramas de las ciencias sociales que le ha sido de más utilidad al ser humano, no solo para conocer el acervo cultural de las diferentes civilizaciones a lo largo del tiempo, sino para permitirle construir una identidad propia. A través de la búsqueda y reconocimiento de hechos históricos, las sociedades han ido incorporando aspectos y acontecimientos de tiempos pasados, como el de la 'Patria Grande', que facilitan la elaboración de su propia identidad. Este es el caso de la obra Historia de la primera República de Colombia, 1819-1831, mi recomendado, ya que, al comprender y desarrollar la
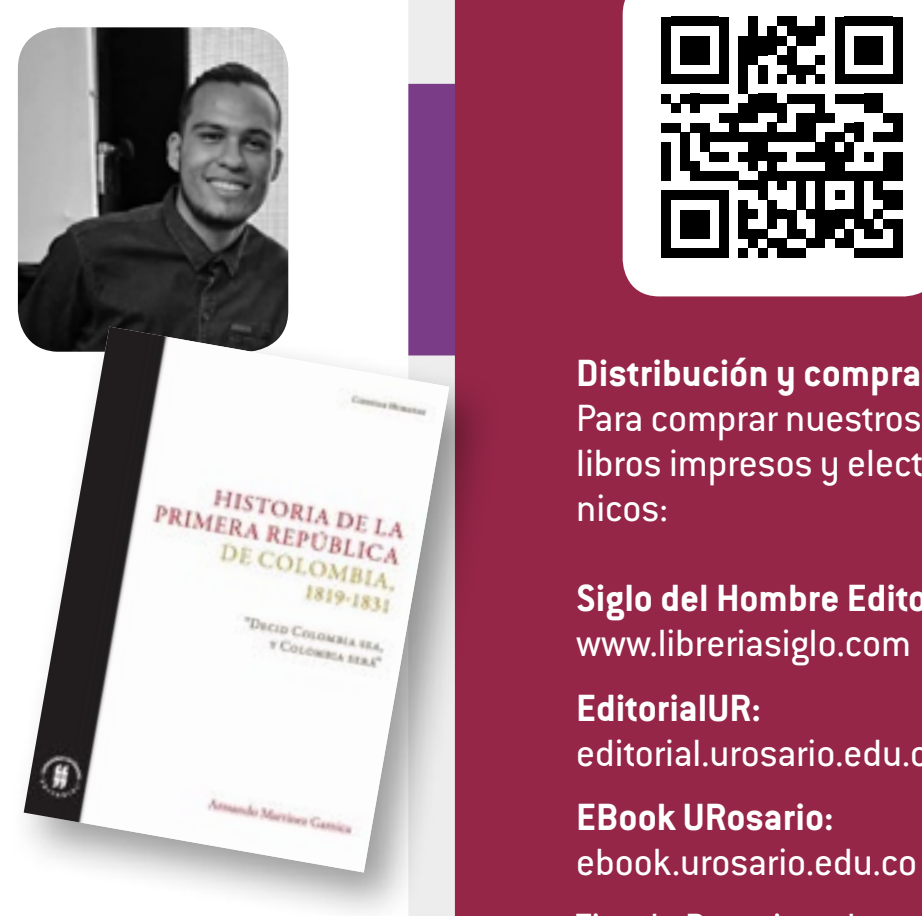
experiencia nacional política de la década de 1820 , los autores contribuyen a la consolidación de identidades de un presente sólido y de un futuro alentador.

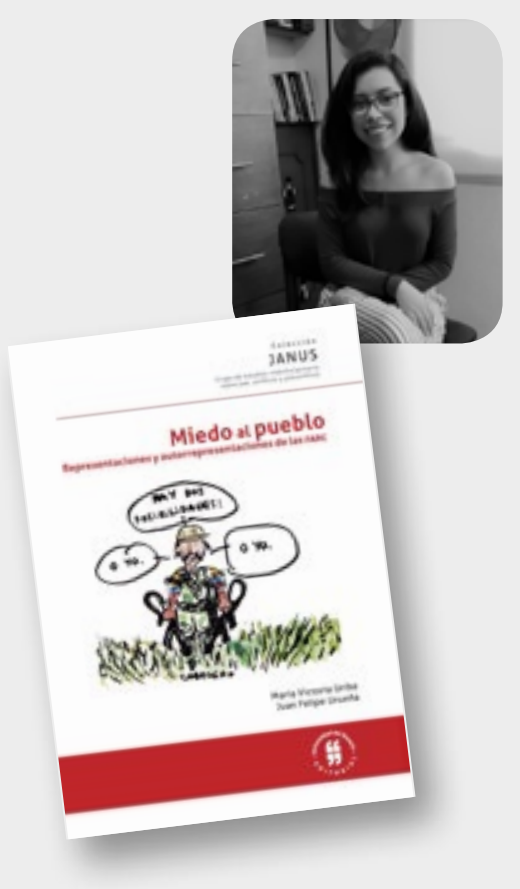

Valentina Caicedo Garzón

Bióloga, Cannon Research Group

Recomendado: Miedo al pueblo. Representaciones

y autorrepresentaciones de las farc

Autores: María Victoria Uribe Alarcón

y Juan Felipe Urueña Calderón

Yo recomiendo el texto Miedo al pueblo. Representaciones y autorrepresentaciones de las FARC a propósito de la situación coyuntural en nuestra nación y la responsabilidad que tenemos como sociedad de conocer $y$ entender, de primera mano, las razones que permitieron que Colombia fuese un país con un conflicto interno de más de 50 años. Creo que estamos en un momento crucial desde que se implementó el proceso de paz en donde las opiniones, muchas veces infundadas, sobre los grupos insurgentes nacen desde los imaginarios. Por esto último, considero que este libro es clave no solo para entender las razones del conflicto, sino para poder hacerlo desde una perspectiva que por lo general siempre se pasa por alto y que permitiría generar una opinión más crítica y objetiva sobre el tema.

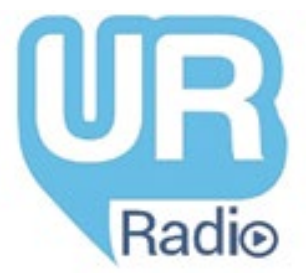

Distribución y compra:

Para comprar nuestros

libros impresos y electrónicos:

Siglo del Hombre Editores: www.libreriasiglo.com

\section{EditorialUR:}

editorial.urosario.edu.co

\section{EBook URosario:}

ebook.urosario.edu.co

Tienda Rosarista de todas las sedes

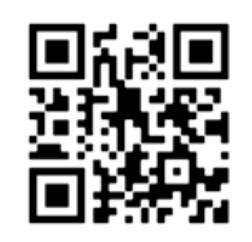

Escucha nuestro programa radial, Las Voces del Libro, en vivo todos los miércoles a las 11 am en URosarioRadio y escucha los podcast de Las Voces del Libro en Spreaker https:// www.spreaker.com/show/ las-voces-del-libro 


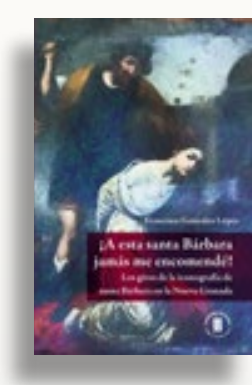

¡A esta santa Bárbara jamás me encomendé!

Los giros de la iconografía de santa Bárbara en la Nueva Granada

Francisco González López P.V.P.: \$ 41.000

P.V.P. digital: \$ 14.000

DOI: https://doi.

org/10.12804/

th9789587844498

ISBN: 978-958-784

449-8

Pág: 188

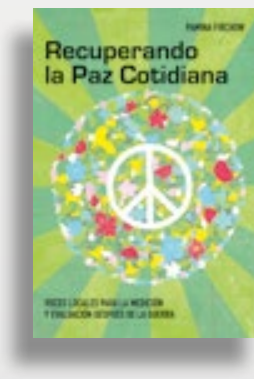

Recuperando la Paz

Cotidiana

Voces locales para la medición y evaluación después de la guerra

Pamina Firchow

P.V.P.: $\$ 60.000$

P.V.P. digital: \$20.000

DOI: https://doi.

org/10.12804/

th9789587844382

ISBN: 978-958-784-

437-5

Pág: 274

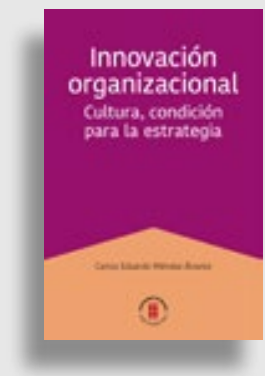

Innovación organizacional.

Cultura condición para la

estrategia

Carlos Eduardo Méndez

Álvarez

P.V.P.: \$ 49.000

P.V.P. digital: \$ 17.000

DOI: https://doi.

org/10.12804/

ta9789587844443

ISBN: 978-958-784-443-6

Pág: 224

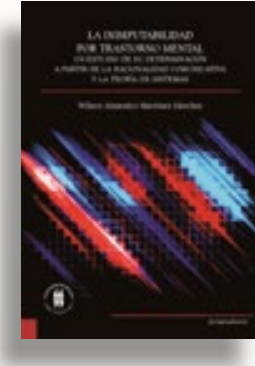

La inimputabilidad por

trastorno mental:

un estudio de su

determinación a partir de la

racionalidad comunicativa

y la teoría de sistemas

Wilson Alejandro Martí-

nez Sánchez

P.V.P.: $\$ 90.000$

P.V.P. digital: $\$ 30.000$

DOI: https://doi.

org/10.12804/

tj9789587843569

ISBN: 978-958-784-

355-2

Pág: 744

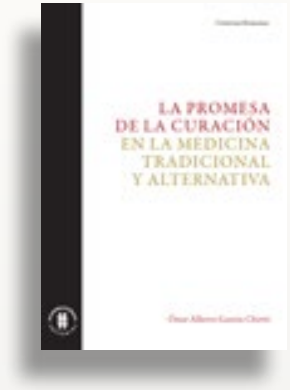

La promesa de la curación en la medicina tradicional y alternativa

Omar Alberto Garzón

Chirivi

P.V.P.: $\$ 50.000$

P.V.P. digital: $\$ 17.000$

DOI: https://doi.

org/10.12804/

th9789587844108

ISBN: 978-958-784-

409-2

Pág: 228

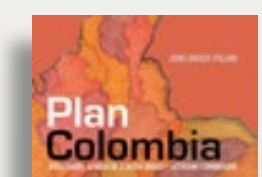

Plan Colombia.

Atrocidades, aliados de Estados Unidos y activismo comunitario

John Lindsay-Poland P.V.P.: \$ 83.000 P.V.P. digital: \$28.000 DOl: https://doi. org/10.12804/ tp9789587844412 ISBN: 978-958-784440-5

Pág: 384

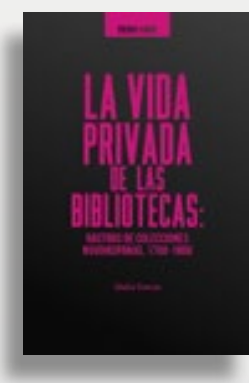

La vida privada

de las bibliotecas: rastros de colecciones novohispanas (1700-1800)

Idalia García

P.V.P.: \$ 132.000

P.V.P. digital: $\$ 44.000$

DOI: https://doi.

org/10.12804/ th978958784318? ISBN: 978-958-784-

318-?

Pág: 606

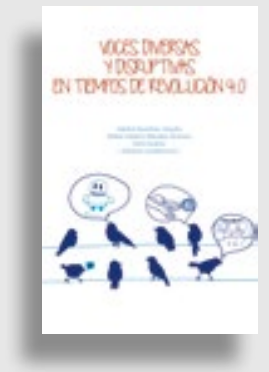

oces diversas y disruptiva en tiempos de Revolución 4.0

Valérie Gauthier-Umaña, Rafael Alberto MéndezRomero, Dora Sánchez (Editores académicos] P.V.P.: \$ 59.000 P.V.P. digital: $\$ 20.000$ DOI: https://doi. org/10.12804/ ti9789587844351 ISBN: 978-958-784434-4

Pág: 270

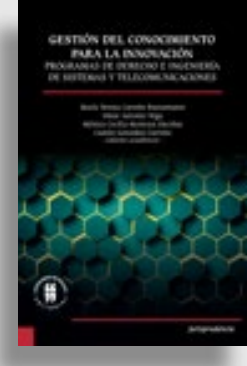

Gestión del conocimiento para la innovación Programas de Derecho e Ingeniería de Sistemas y Telecomunicaciones

María Teresa Carreño Bustamante, Omar Antonio Vega, Mónica Cecilia Montoya Escobar, Camilo González Carreño (Editores académicos] P.V.P.: \$ 59.000 P.V.P. digital: $\$ 20.000$ DOI: https://doi. org/10.12804/ tj9789587843958 ISBN: 978-958-784394-1

Pág: 296 